\title{
QUESTIONNEMENTS AUTOUR DE LA GESTATION POUR AUTRUI
}

\section{Karine Bréhaux, Béatrice Delépine-Panisset}

Éditions du Cerf | «Revue d'éthique et de théologie morale »

$2014 / 5 n^{\circ} 282$ | pages 37 à 53

ISSN 1266-0078

ISBN 9772204352834

Article disponible en ligne à l'adresse :

https://www.cairn.info/revue-d-ethique-et-de-theologie-morale-2014-5-page-37.htm

Distribution électronique Cairn.info pour Éditions du Cerf.

(C) Éditions du Cerf. Tous droits réservés pour tous pays.

La reproduction ou représentation de cet article, notamment par photocopie, n'est autorisée que dans les limites des conditions générales d'utilisation du site ou, le cas échéant, des conditions générales de la licence souscrite par votre établissement. Toute autre reproduction ou représentation, en tout ou partie, sous quelque forme et de quelque manière que ce soit, est interdite sauf accord préalable et écrit de l'éditeur, en dehors des cas prévus par la législation en vigueur en France. Il est précisé que son stockage dans une base de données est également interdit. 


\section{QUESTION NEMENTS AUTOUR DE LA GESTATION POUR AUTRUI}

\section{N T R O D U C T I O N}

Les débats autour de la naissance suscitent de vifs mouvements sociaux. Ils interpellent les communautés politiques et scientifiques sur les limites à définir dans l'encadrement des pratiques relatives à la naissance. Les difficultés des débats bioéthiques relatifs à la naissance ont amené la société civile à faire intervenir le législateur. On demande l'avis à l'homme de loi, pas toujours prêt à traiter des questions d'une telle complexité à un niveau interdisciplinaire. D'autres débats sociaux tels que l'avortement ont montré par le passé le besoin de "temps " pour démêler les affaires et obtenir un consensus social. Il s'ensuit des débats politiques et bioéthiques passionnés.

Qu'en est-il du droit de naître dans les domaines de la procréation et du début de la vie? Il semble que l'on puisse en tout état de cause décider de la vie ou de la mort d'un embryon. Il est possible d'apprécier qualitativement la vie d'un embryon ou d'un foetus selon des critères et des situations particulières.

Le droit de naître pose la question du statut juridique de l'embryon et de l'usage social qu'il pourrait en être fait. Deux tendances éthiques se dégagent sur le statut juridique et métaphysique de l'embryon : une tendance déontologique qualifiant le droit de disposer de l'embryon comme illégitime du fait de la primauté du respect de l'individu, et une tendance téléologique qualifiant le droit de disposer de l'embryon comme légitime du fait de la primauté du bien-être et des désirs humains. Les 
risques de dérives sont présents. La potentialité du matériel biologique de l'embryon, l'instrumentalisation du corps féminin et la réification de l'être humain sont sans cesse menacées. Les principes d'inviolabilité de la personne et de la non-commercialisation du corps humain sont en permanence en danger.

Le débat français autour de l'affaire Nicolas Perruche a mis en valeur la volonté de reconnaître un nouveau droit : le droit à ne pas naître handicapé.

L'éthique n'est ni une science, ni un système institutionnel de règles, ni un savoir-faire. Cependant, sa nécessité nous oblige à la concevoir comme un objet de savoir et à fonder l'agir moral sur des principes dits éthiques. L'éthique entendue comme sagesse ordonnée à l'action est ce qui guide les choix, les décisions, délibère sur des buts, des perspectives d'action.

L'estimation de la qualité de vie d'un être humain est un enjeu juridico-éthique. Il pose la question de l'interventionnisme légitime ou illégitime d'instances de contrôles légales, institutionnelles et médicales dans l'élaboration de normes sociales liées à la reproduction sexuelle :

"La reconnaissance d'un droit à l'enfant à ne pas naître dans certaines conditions apparaîtrait hautement discutable sur le plan du droit, inutile pour assurer l'avenir matériel des personnes souffrant de handicaps congénitaux et redoutables sur le plan éthique. En effet, un tel droit risquerait de faire peser sur les parents, les professionnels du diagnostic prénatal et les obstétriciens, une pression normative d'essence eugénique. " ${ }^{1}$

C'est en 1984, que le Comité Consultatif National d'Éthique emploie, à propos de l'embryon, le terme de "personne potentiellement humaine». Le Comité Consultatif National d'Éthique reprend la conception aristotélicienne de la personne. Chez Aristote, l'embryon entre dans la conception d'une personne humaine en puissance, dont l'être est en train d'advenir, de se constituer, de se développer. L'achèvement de l'être humain sera effectif au terme de son développement physique et psychologique, et dans le langage aristotelicien sera dit "en acte ". C'est donc en vue de l'actualisation de la personne définie comme

1. Cahier du CCNE, avis numéro 68 sur Handicaps congénitaux et préjudice, numéro 104, Paris, 29 mai 2001. 
finie que les efforts de l'être en puissance se tournent dès ses premières semaines d'existence.

L'embryon, dès la fécondation, est une personne potentielle en ce qu'il est en puissance. Actuellement, en France, la recherche ne porte que sur des embryons issus de couples suivis en infertilité ou en programme de Diagnostic Pré Implantatoire. Aucun embryon n'est créé pour la recherche (loi de bioéthique de juillet 2011 et loi autorisant la recherche sur les embryons du 16 juillet 2013).

Après avoir fait état des questions relatives aux pratiques médicales et sociales de la Gestation Pour Autrui, nous montrerons que philosophiquement cette controverse sociétale puise ses origines dans une problématique plus générale, celle de l'identité personnelle.

\section{QUESTIONNEMENT}

\section{Sur La Gestation Pour Autrui}

\subsection{La Gestation Pour Autrui : pratique technique et dilemme éthique}

La demande d'un couple faisant appel à une autre femme pour porter leur enfant, n'est pas sans reconfigurer le schéma familial habituel et de fait, interroge certains, dérange d'autres. Rappelons que sous le terme Gestation Pour Autrui, deux entités se distinguent : il s'agit soit d'une Fécondation In Vitro avec les gamètes du couple d'intention et transfert embryonnaire chez la gestatrice, soit d'insémination intra-utérine avec le sperme du père d'intention et, dans ce cas, la gestatrice est également la génitrice. Sur le plan purement scientifique, il s'agit de variantes des techniques d'Assistance Médicale à la Procréation (AMP) classiques.

Mais sur le plan éthique, l'intention est plus complexe et ce qui peut être qualifié comme un progrès thérapeutique pourrait ouvrir une voie préalablement inenvisageable. Et en écho à cette proposition, de se dire : "si c'est possible, j'y ai droit " (droit-créance). Mais rien ne justifie qu'une évolution scientifique puisse satisfaire les désirs d'un individu sous prétexte que c'est techniquement possible, qu'il faut coller aux mentalités qui évoluent et que le projet s'inscrit dans un cadre médical. 
À ce jour, les indications médicales susceptibles de relever d'un recours à ce mode de conception sont extrêmement rares (développement incomplet ou absence congénitale d'utérus, perte de fonctionnalité utérine d'origine iatrogène). Quant aux indications non médicales, sociétales telles que le recours d'un couple d'hommes à la Gestation Pour Autrui (faisant intervenir une ou deux femmes, l'une donnant ses ovules fécondés in vitro par les gamètes d'un des deux hommes puis transfert embryonnaire dans l'utérus de la même femme ou dans celui d'une deuxième femme), elles pourraient faire suite à l'autorisation d'accès à tout type d'AMP pour les couples de femmes, ceci dans un souci d'égalité.

Concernant la Gestation Pour Autrui pour les couples hétérosexuels, quels pourraient être les intérêts et les risques de chacun - enfant, parents d'intention, mère porteuse - dans le contexte particulier de la Gestation Pour Autrui?

\subsection{La Gestation Pour Autrui, entre questions de droit et d'accès aux soins}

Le couple susceptible de se tourner vers une demande de Gestation Pour Autrui et plus particulièrement la femme, souffre d'infertilité pour laquelle il n'est pas possible de proposer une thérapeutique classique - inséminations artificielles, Fécondation In Vitro. Cette grossesse inaccessible et la frustration qu'elle engendre, créent une inégalité d'accès à l'enfantement qui peut, pour certaines femmes, devenir difficilement acceptable. Cette femme qui a reçu la vie se trouve dans l'incapacité de la donner. Ce corps pourtant féminin est amputé, il ne sera jamais le vecteur de la grossesse, il est définitivement privé de la maternité et de l'enfantement. La Gestation Pour Autrui rend possible une procréation en l'absence du corps de la mère d'intention, celle-ci s'effaçant transitoirement du temps de la grossesse et de la naissance de son enfant. Comment trouver son rôle de mère nourricière, alors qu'elle ne porte pas l'enfant? Malgré cette mise à l'écart imposée, va-t-elle veiller, surveiller ou "couver" cette gestation sans trop d'intrusion et en toute bienveillance? Le recours à la Gestation Pour Autrui n'a, de toute évidence, rien à voir avec l'adoption pour laquelle il s'agit d'accueillir un individu déjà conçu au sein d'un couple inconnu, enfant "à la recherche " d'un accueil par des parents adoptants ; ces derniers 
n'étant pas obligatoirement stériles et surtout, c'est bien l'enfant privé de famille qu'il faut secourir et accompagner. De plus, dans les pays où la Gestation Pour Autrui est pratiquée, il n'y a pas d'anonymat de la mère porteuse ce qui risque de créer chantage et pressions entre elle et le couple en demande; ce dernier peut se sentir redevable vis-à-vis d'elle d'où, là aussi, la possible notion de dette. Dans ces pays autorisant la Gestation Pour Autrui, les mères porteuses sont reconnues comme ayant des droits en regard de leur état de grossesse (prise en charge des frais médicaux, droit de garder l'enfant, à des dédommagements...)

\subsection{La Gestation Pour Autrui : un acte généreux non sans risque?}

La contribution de la gestatrice à donner la vie est louable si elle est dénuée de tout intérêt financier. La Gestation Pour Autrui crée-t-elle un préjudice nécessitant un dédommagement, une compensation sans rémunération? Si l'acte s'inscrit comme le maillon d'une chaîne de vie, sans pression financière, la notion de marchandisation s'amenuise. Par ailleurs aucune grossesse ni aucun accouchement n'est sans risque : le consentement est-il total et a-t-elle, en toute conscience, mesuré le risque d'atteinte à l'intégrité de son corps? Comment se situe le geste de la femme qui porte l'enfant et va le remettre au couple demandeur en se mettant à son service et qu'en est-il de sa dignité ? Quelle valeur revêt cet acte, y a-t-il une élaboration psychique particulière? Est-ce par pure solidarité pour le bénéfice d'autrui ? Est-ce une dette? Est-ce dans l'espoir d'une valorisation narcissique? Quels éléments motivent le projet de cette femme vis-à-vis de cet enfant avec lequel elle aura partagé une partie de vie et de son sang mais dont elle ne sera jamais la maman? Peut-être va-t-elle devoir renoncer à devenir la mère de cet enfant (si la gestatrice n'abandonne pas l'enfant à la naissance, elle est, de principe, la mère de l'enfant). D'autre part, comment va-t-elle préserver sa vie privée, reste-t-elle autonome? Est-elle totalement libre de son choix?

Cet enfant n'est pas le sien mais elle le porte : dans ce rôle matriciel provisoire, comment cette femme va-t-elle investir la grossesse? La valeur symbolique de la maternité n'est-elle pas fragilisée? La traduction anglo-saxonne de mère porteuse 
est "suroggate mother" ou mère de substitution; c'est un peu comme s'il fallait "prendre la place de ", combler un manque, se substituer au vide laissé par la mère d'intention incapable de remplir ses fonctions. Cette femme tente de pallier le déficit d'une autre, de réparer cette perte. Remplacer des compétences à jamais perdues, le temps d'une grossesse, et pourquoi pas? La relation dissymétrique fondée sur la toute-puissance de la gestatrice désireuse de porter secours place la mère d'intention en situation de vulnérabilité, d'attente et de dévalorisation.

Afin de se préserver et pour sortir de cette expérience, indemne psychiquement, la femme qui va porter l'enfant, doitelle tenter, à son corps défendant, de mettre entre parenthèses ces neuf mois? Faire, en quelque sorte, l'économie d'éventuelles projections et de ce qui se joue en elle. Parviendra-t-elle à se mettre à distance et à quel prix? Quelle devrait être alors "la juste distance", être à côté de la personne et non "à la place de». Et si elle veut se protéger et anticiper cette séparation obligatoire, elle devra tout mettre en ouvre pour effacer les interactions et les liens de la dyade foutus/mère tissés pendant cette vie à deux. Relation interrompue dès les premiers instants de vie extra-utérine de l'enfant, cet enfant qu'elle ne nommera pas. S'agit-il, comme le soulignent M. Szejer et JP Winter d'un abandon sur ordonnance :

" on oublie que la prescription de fécondation in vitro nécessaire à cette GPA cautionnée par la science équivaut à une ordonnance d'abandon ${ }^{2}$ "?

De plus, dédier ce corps (en prenant le risque de réduire le dualisme cartésien à la seule présence du corps réifié) à la gestation ne risque-t-il pas de réduire cet acte à un simple "moyen" puisque pour cette femme, de passage dans la vie de cette autre famille, il n'y aura pas de suite donc pas de "fin "? Le développement de l'individu démarre dès sa conception et est modelé par de multiples interactions au sein de l'utérus maternel et par le biais du placenta. On peut donc supposer que l'environnement extérieur durant la grossesse aura un impact sur le développement post-natal de ce dernier. Et les influences environnementales ainsi que le comportement maternel évoluent

2. SZEJER et JP WINTER "Abandon sur ordonnance" Tribune publiée le 23.07.08 dans Libération 
parallèlement à la marque du code génétique et aux empreintes épigénétiques constituant le sujet. De même, chacun sait que le vécu émotionnel n'est pas sans conséquence sur le foetus, en effet selon Emmanuel Sapin :

"un état douloureux ressenti par la femme enceinte est un stress auquel elle réagit en sécrétant des hormones (adrénaline et cortisol) qui auront un effet sur le cerveau du foetus dès le deuxième trimestre de la grossesse, avec des conséquences sur le comportement post-natal de l'enfant ${ }^{3}$."

Et de s'interroger sur les conséquences du mode de vie de la mère porteuse (ex : tabagisme, alcoolisme...) : qui est responsable d'éventuelles séquelles portées par l'enfant? Le couple d'intention pourrait légitimement s'octroyer un droit de regard sur tout élément intentionnel et maîtrisable susceptible de troubler cette grossesse puisqu'il s'agit de leur enfant. D'où une possibilité de conflit et de procès en cas de handicap de l'enfant. Quant au congé maternité, la Cour de Justice de l'Union Européenne a été saisie pour une affaire dans laquelle un congé maternité était réclamé par la mère d'intention (communiqué de presse numéro 115/13, Luxembourg, 26.09.2013, conclusions de l'avocat général dans l'affaire C-167: 12CD/ST, page 9).

\subsection{La place de l'enfant à naître}

Et l'enfant, que retiendra-t-il de sa vie intra-utérine et de sa naissance, hors du corps de sa mère? Comment se construira son histoire, son identité? L'enfant n'est pas un dû, il ne doit pas être chosifié. Doit-il ou peut-il rejeter tout élément d'attachement précoce? Qui sont ses parents et comment définir la place de chacun? Combien a-t-il de maman(s), qui est son père (si recours à un donneur de sperme), les enfants de la mère qui l'a porté, qui sont-ils vis-à-vis de lui ? Selon Geneviève Delaisy de Parseval,

" la Gestation Pour Autrui donne un sens nouveau aux définitions mêmes de père, mère, frère, sœur, enfant, sibling, oncle, tante, grands-parents qui n'ont plus désormais de contenus fixes ${ }^{4}$ ».

3. Sapin Emmanuel, www.génèthique.org. Lettre mensuelle de septembre 2013 : "Le foetus est sensible à la douleur... et aux émotions ".

4. Geneviève Delaisy De Parseval «La naissance. Histoire, culture et pratiques d'aujourd'hui "sous la direction de René Frydman et Myriam Szejer. Éd Albin Michel, p. 397. 
Quoi qu'il en soit, en l'absence de repères clairs de filiation, comment cet enfant va-t-il pouvoir trouver une place qui soit la sienne? L'équilibre psychoaffectif est-il réellement en danger ou, au contraire, faut-il dédramatiser considérant que cet enfant saura s'adapter? La réponse étant directement liée à la casuistique, les deux suggestions sont sans doute recevables. Les publications britanniques telles que celles de Golombock ${ }^{5}$ ne semblent pas mettre en évidence de problème particulier, dans le premier âge. De quel droit lui imposer une venue au monde dans des conditions où gestation et filiation sont dissociées? Enfin, conformément à la loi actuelle, quels sont les droits de cet enfant conçu à l'étranger et ramené sur le sol français? Comment se situe la notion d' "intérêt supérieur de l'enfant" (article 3 de la Convention relative aux Droits de l'Enfant, 1959)? Quant aux enfants de la mère porteuse, que pourront-ils s'imaginer en voyant que leur propre mère, celle qui leur a donné la vie, est capable de porter un enfant et s'en séparer dès sa naissance? Le fantasme d'abandon n'est peut-être pas loin (et l'enfant de se demander : "Pourquoi certains restent et d'autres partent de chez nous?»). Les repères structurants et l'équilibre familial peuvent-ils être mis en danger?

\section{QuelQues PISTES DE RÉFLEXIONS}

\subsection{Les risques de dérives identifiables}

Les enjeux non négligeables soulevés par la question de la grossesse pour Autrui bousculent et invitent à la réflexion. Le faible nombre de publications sur le sujet contraste avec la multitude de sites internet vantant les mérites des agences mettant en relation les gestatrices potentielles et les couples candidats à la Gestation Pour Autrui : là où le business est roi, y a-t-il risque d'exploitation de femmes défavorisées? Et même si les pratiques clandestines restaient limitées, comment être bienveillant dans le respect de la dignité humaine, vis-à-vis de l'enfant à naître, du couple d'intention, de la femme qui portera l'enfant, son conjoint et leurs enfants? Et au nom du principe d'indisponibilité

5. Golombock et al Dev Psych 2004, 40, 400-411; Journ Child Psycho Psychiatrie 2006, 47, 213-222; Hum Reprod 2006, 21, 1918-1924. 
du corps humain, il semble légitimement souhaitable de lutter contre une marchandisation de toute partie du corps humain.

Le recours à la Gestation Pour Autrui ne peut être réduit à une thérapeutique palliative dans l'optique de suppléer à un dysfonctionnement organique. Pour autant, la détresse, au combien recevable, des femmes souffrant d'une stérilité irréversible associée à l'absence d'alternative thérapeutique doit être prise en compte et très étroitement accompagnée. Mais faut-il faire fi de l'intérêt de l'enfant au profit de celui du couple? A contrario, peut-on se convaincre que "l'on naît comme on est " (dispositions naturelles différentes entre les individus) et qu'il faut accepter ce redoublement d'inégalité (naturelle et médicale) de prise en charge de la maladie (la greffe d'utérus et l'utérus artificiel ne sont pas à l'ordre du jour mais permettrait de réduire les inégalités)? Pour certains, sa propre vulnérabilité face à la maladie peut générer un sentiment de frustration insupportable, un dû dont l'intensité va croissant avec l'évolution des prouesses médicales, ce qui est ressenti comme une injustice commande l'injonction de réparer le préjudice. Comment, dans ce contexte de fragilité amère, parvenir à se faire violence en se mobilisant pour tenter, malgré tout, d'accepter ce constat et de vivre tel que l'on est en tentant de donner un autre axe au projet parental ? Comment renoncer à l'idée d'enfantement?

Actuellement, des couples se rendent à l'étranger en vue de la réalisation d'une Gestation Pour Autrui illicite. Or, la filiation à l'égard de la mère d'intention ne peut se faire puisque celle-ci ne peut être établie que vis-à-vis de la femme qui accouche. Comment sortir de cette impasse sans bâtir un droit objectif sur un droit subjectif en bricolant une filiation en marge? Faut-il cautionner un droit à l'enfant (droit-créance, avoir le droit à...) afin de restaurer une égalité sociale entre tous les couples?

L'examen soigneux des conséquences que son autorisation pourrait engendrer sous prétexte de s'aligner sur un fait accompli adopté par d'autres états, doit impérativement mettre en balance les intérêts de chacun : est-on efficace, bienfaisant et respecte-t-on les droits de chacun? Autrement dit, la Gestation Pour Autrui constitue-t-elle le moyen thérapeutique le plus adapté pour traiter la pathologie de la mère d'intention sans malfaisance envers chaque protagoniste? Ne courons-nous pas le risque à privilégier le droit du couple à l'enfant sur l'intérêt premier de l'enfant à 
naître? Comment une femme peut être amenée à considérer son corps comme un moyen (même à des fins altruistes) et non une fin? Comment préserver, protéger la femme qui en situation de vulnérabilité (contraintes d'un tiers, précarité) peut se trouver dans une situation non voulue? Comment éviter la confrontation entre une logique marchande et une logique juridique de défense de l'intérêt de l'enfant? Est-ce que la voie de dépénalisation de la pratique de la Gestation Pour Autrui en France peut s'avérer une piste de réflexion? Les conditions de réalisation de la Gestation Pour Autrui pourraient-elles être un jour "débarrassées » de toute problématique éthique?

\subsection{Quelles prises de positions sont observées en France?}

Le législateur est aux prises avec des débats passionnés et des pressions sociétales diverses. Cependant, il construit un cadre dont le fondement doit s'appuyer sur le respect de la dignité humaine. Il doit rester en dehors des affects des uns et des autres afin de cheminer dans le domaine du raisonnable sans nuire à quiconque. Le dernier avis rendu par le Comité National d'Éthique pour les Sciences de la Vie et de la Santé en 2010 était favorable au maintien de la législation en vigueur.

Derrière ces questions, quelles options sont disponibles, envisageables dans une société culturelle où la Gestation Pour Autrui n'est pas légalisée? À la lecture du rapport Filiation, origines, parentalité : le droit face aux nouvelles valeurs de responsabilité générationnelle (2014), mené par Irène Théry, on comprend que des raisons historiques, culturelles et juridiques nourrissent ce positionnement réglementaire français. Ce groupe de travail défend

"le sens d'une réforme de la filiation qui doit faire coexister à égale dignité trois modalités d'établissement de la filiation : l'engendrement par procréation charnelle, l'adoption, l'engendrement avec tiers donneur ${ }^{6}$.

Il recommande de dépasser le modèle matrimonial de filiation instaurant une asymétrie entre les sexes et une hiérarchie

6. Rapport Filiation, origines, parentalité : le droit face aux nouvelles valeurs de responsabilité générationnelle, Irène Théry, Ministère des Affaires Sociales et de la Santé, 2014, page 18. 
complète entre la maternité et la paternité. Il se prononce en faveur d'une conception de la famille contemporaine ouverte aux pluriparentalités avec des "parents " au sens de la filiation ayant une place dans l'histoire et l'identité personnelle de l'enfant, des parents de naissance, des donneurs de sperme ou d'ovocyte, beaux-parents.

Actuellement, face à une demande sociale de plus en plus forte, on échange sur la question de la filiation : qu'est-ce qu'un parent? Quels sont les droits, les devoirs, les interdits qui caractérisent ce statut? Deux options se présentent : maintenir le droit en l'état et le système de valeurs et de représentations actuels ou réformer dans le sens d'un droit ouvert à tous et pluraliste et accepter un nouveau référentiel.

En Italie, une femme est actuellement enceinte de deux jumeaux qui ne sont pas biologiquement les siens (erreur médicale d'implantation d'embryon lors de la fécondation in vitro) A qui appartiendront les jumeaux à la naissance? À la mère porteuse? Aux parents biologiques? La question de l'identité première est posée. Le milieu influence-t-il ou pas le développement de l'être? Il semble qu'il n'y ait pas de réponse, et que les champs du possible restent ouverts.

La disparité des conditions socio-économiques entre les pays du Nord (les pays d'ex Europe de l'Est par exemple) et du Sud (entre autre la Californie) et la possibilité de "louer son ventre" ne peut être tenue à l'écart de ces questionnements.

D'un point de vue éthique, il faut éviter que la situation de mère porteuse ne soit subie et qu'elle adhère pleinement à ce type d'engagement. Dans cette même perspective, il faut aussi éviter une double sanction pour des couples en demande d'enfant : une sanction biologique (impossibilité de mettre au monde un enfant, "d'enfanter") et une sanction sociale (le coût du recours à une Gestation pour Autrui). Dans le rapport d'information "Contribution à la réflexion sur la maternité pour autrui " (2008) mené par Alain Milon, Henri de Richemont, et Michèle André, il est proposé d'autoriser et d'encadrer la Gestation Pour Autrui sous des critères sociaux et médicaux déterminés. Dans cette logique, se pose la question de la détermination du caractère exceptionnel d'une situation vécue par rapport à une autre. Ce débat autour de "l'exceptionnalité de la situation donnant droit à...", a déjà été pointé du doigt sur d'autres controverses comme celle de 
la fin de vie et "l'exceptionnalité de l'acte d'euthanasie». Il est difficile de demander au corps juridique ou au corps médical de distinguer les situations appartenant à l'ordre de l'exceptionnalité et celles qui restent du domaine de "l'ordinaire».

\section{POINT DE VUE PHILOSOPHIQUE}

\subsection{La Gestation Pour Autrui : une question contemporaine de sociologie et de philosophie politique}

On ne peut pas présenter une vision séparée du politique et de l'éthique, en considérant l'Assistance Médicale à la Procréation et/ou la Gestation Pour Autrui uniquement comme des problèmes de santé publique. Bien au contraire, ces techniques médicales sont formalisées comme des enjeux de santé publique au nom de l'intérêt général. La question n'est donc pas posée au politique lorsque les pratiques sociales et les techniques médicales liées au droit à l'enfant ont quitté l'espace politique pour le huis clos des comités d'éthique. Mais, l'histoire a déjà montré que la science était elle-même une question par essence politique, car elle constitue un enjeu de pouvoir. La recherche du consensus et du compromis en matière des droits de naître et de l'enfant, prônée par le libéralisme politique, conduit à des projets biopolitiques différents. Ces consensus déterminent la place à accorder à de nouveaux droits individuels et aux libertés publiques.

D'un point de vue sociologique, est-ce que toute demande déviante aux yeux de certains par rapport à la norme doit être médicalisée? Qui fixe la norme? Et pour quelles raisons?

Une des leçons tirées de nos époques de guerres est sûrement l'urgence de concevoir un monde où chacun possède des droits et une valeur propre, égale aux autres et cela doit nous donner le sentiment d'appartenir à une communauté. Le revers de cette conception est un individualisme exacerbé et la quête de la reconnaissance de droits nouveaux.

\subsection{La question philosophique de l'identité personnelle}

La problématique centrale de cette controverse sociétale est celle de l'identité personnelle. La question de l'identité personnelle a été longuement traitée dans la littérature philosophique et scientifique - pensons à Hobbes, Locke, Descartes, Hume et 
Kant par exemple. Plus proches de nous, nous avons les œuvres de Paul Ricoeur comme références incontournables.

Locke définit l'identité personnelle dans Essai concernant l'entendement bumain (livre 2, chapitre 27). L'auteur insiste sur le fait que le siège de l'identité personnelle est la conscience :

"C'est dans la conscience seule que consiste l'identité personnelle... et aussi loin que cette conscience peut s'étendre sur les actions ou pensées déjà passées, aussi loin s'étend l'identité de cette personne, le moi est présentement le même qu'il était alors, et cette action passée a été faite par le même moi que celui qui se le remet à présent dans l'esprit? . "

Selon Locke, l'identité personnelle est liée à la mémoire. Le questionnement scientifique actuel porte sur la faculté de sentir et de se souvenir du foetus au cours de sa vie intra-utérine. Si c'est le cas, dans quelle mesure cet ensemble de connaissances sensibles contribue-t-il à la formation de son identité personnelle? Cette liaison entre la mémoire et la conscience est bien sûr critiquée par d'autres auteurs. Cette conception de l'identité personnelle par Locke renvoie aux préoccupations actuelles autour des enjeux identitaires de la Gestation pour Autrui ou de la greffe et de la transplantation d'organes. Ces problèmes engendrés par une définition unanime de l'identité personnelle posent la nature de la personne humaine. Ces "puzzling $\operatorname{cases}^{8}$ » sont critiquées par Paul Ricoeur, qui lui soutient l'importance de l'historicité de l'individu dans la construction de son ipséité.

La tradition empiriste, dont l'un des grands fondateurs est Hume, soutient la faculté de sentir au fondement de l'identité personnelle. Dans le Traité de la Nature humaine (livre 1, partie 4, section 6) Hume écrit :

"Un vaisseau dont une partie importante a été modifiée par de fréquentes réparations est toujours considéré comme le même vaisseau, et la différence des matériaux ne nous empêche pas d'attribuer de l'identité. La fin commune à laquelle tendent les parties est la même sous toutes leurs variations et elle occasionne une transition aisée de l'imagination d'un état du corps à un autre?".

\footnotetext{
7. Locke, Essai concernant l'entendement humain, livre 2, chapitre 27, Paris, éditions Broché, 2004.

8. "Puzzling cases" renvoie à la question de l'intégration d'éléments humains d'une personne sur une autre personne et ses conséquences sur l'identité première.

9. Hume, Traité de la Nature bumaine, livre 1, partie 4, section 6, Paris, éditions Poche, 1999.
} 
Pour Hume, c'est à partir de sensations particulières que l'on peut construire le moi.

Nous sommes à nouveau dans l'impasse : est-ce que les sensations perçues au cours de la grossesse par l'embryon ou le foetus sont constitutives des propriétés du soi?

\section{CONCLUSION}

La Nature fait-elle bien les choses? Expression courante témoignant de la bienveillance de la Nature sur le cours des événements, de nos vies. C'est aussi reconnaître une autorité de la Nature sur le cours des choses. Quelle part d'arbitraire y a-t-il dans cet ordre apparent et quel impact du progrès technique? Que dire si nous intervenions nous-mêmes, le genre humain, sur le cours de la Nature? Cherchons-nous à l'égaler dans ses inventions? Dans l'Éthique ${ }^{10}$, Spinoza définit la Nature ou Dieu comme Être nécessaire dont découle et dont dépend l'existence d'autres êtres. La Nature est un sujet souverain et réside en chacun de nous. L'homme, sujet nouménal et représentatif, se voit assigner la tâche de comprendre l'ensemble de ces manifestations naturelles. Peut-on alors parler d'un dessein de la Nature, qui s'évertue à faire évoluer les capacités de l'entendement humain? Il est de la nature des principes de l'entendement humain que de "donner une forme" à la Nature. L'homme peut-il espérer se détacher de "ce point de vue obligé imposé par la Nature "?

Cette prouesse technique de la Gestation Pour Autrui n'est-elle pas l'illustration de ce jeu inconscient entre l'Homme et la Nature?

$$
\text { Karine BREHAUX }
$$
Philosophe et Docteur en science politique, Sciences-Po Paris.

$$
\text { Dr Béatrice DELÉPINE-PANISSET }
$$

Service de Génétique et Biologie de la Reproduction - CECOS Hôpital Maison Blanche CHU de Reims.

10. Spinoza, L'Éthique, partie 1, Paris, éditions Points Seuil, page 15, 1999. 


\section{Bibliographie}

Textes réglementaires

- Agence de la biomédecine - Étude comparative de l'encadrement juridique international - octobre 2008.

- Arrêt n 1285 du 17 décembre 2008 Cour de cassation - Première chambre civile.

- Cahiers du C.C.N.E., Avis numéro 68 sur Handicaps congénitaux et préjudice, numéro 104, Paris, 29 mai 2001.

- Cour Européenne des Droits de l'Homme, 6 octobre 2005, Draon c/France et Maurice c/France (requêtes n ${ }^{\circ} 151303$ et $n^{\circ}$ 11810/03).

- Cour Européenne des Droits de l'Homme, 6 octobre 2005, Draon c/France.

- Convention de New York relative aux droits de l'enfant, 20.11.1989.

- Décision n 94-343/344 DC du 27 juillet 1994 par le Conseil Constitutionnel sur la loi relative au respect du corps humain et loi relative au don et à l'utilisation des éléments et produits du corps humain, à l'assistance médicale à la procréation et au diagnostic prénatal.

- Rapport d'information fait au nom de la commission des Affaires sociales et de la commission des Lois constitutionnelles, de législation, du suffrage universel, du Règlement et d'administration générale par le groupe de travail sur la maternité pour autrui, Michèle ANDRÉ, Alain Milon et Henri de Richemont, Sénat, Paris, 25 juin 2008.

- Rapport d'information fait au nom de la commission des Affaires sociales et de la santé, Ministère délégué chargé de la famille, Irène Thery, Anne-Marie Leroyer sur «Filiation, origines, parentalité, le droit face aux nouvelles valeurs de responsabilité générationnelle », 2014.

- Rapport d'information fait au nom de la mission d'information pour la révision des lois de bioéthique, Paris : Assemblée Nationale.

- Rapport du comité de réflexion sur le préambule de la Constitution, Redécouvrir le préambule de la Constitution, remis au Président de la République, décembre 2008.

Ouvrages et articles

- Delaisy de Parseval, "La naissance. Histoire, culture et pratiques d'aujourd'hui "sous la direction de René FrYDMan et Myriam SzEJER. Éd Albin Michel, p. 397

- Golombock et al DEV PSYCH 2004, 40, 400-411; Journ Child Psycho Psychiatrie 2006, 47, 213-222 ; Hum Reprod 2006, 21, 1918-1924.

- HUME, "Traité de la Nature humaine", livre 1, partie 4, section 6, Paris, éditions Poche, 1999.

- Locke, "Essai concernant l'entendement humain ", livre 2, chapitre 27, Paris, éditions Broché, 2004.

- SpINOZA, "L'Éthique», partie 1, Paris, éditions Points Seuil, page 15, 1999.

\section{Index}

- AMP : Assistance médicale à la procréation. Ensemble des techniques médicales permettant à un couple infertile de procréer. On distingue 
classiquement les techniques intraconjugales dans lesquelles la procréation se passe dans le couple et les techniques avec tierce personne : donneur de gamètes ou gestatrice.

- CCNE : Comité consultatif national d'éthique.

- CNCDH : Commission Nationale Consultative des Droits de l'Homme.

- CPDPN : Centres pluridisciplinaires de diagnostics prénataux.

- DO-GPA : Don d'ovocyte-Gestation pour autrui. L'enfant est conçu à partir des ovocytes d'une femme donneuse, fécondés in vitro par le sperme du père intentionnel; les embryons ainsi obtenus sont replacés dans l'utérus de la gestatrice. Il n'existe donc aucun lien génétique entre l'enfant et la mère gestationnelle.

- DPI : Diagnostic préimplantatoire.

- DPN : Diagnostic prénatal.

- FIV : Fécondation in vitro. Technique d'assistance médicale à la procréation qui consiste à prélever les ovocytes au niveau des ovaires de la femme, les féconder in vitro avec le sperme du mari puis replacer le ou les embryons obtenus directement dans l'utérus de la femme (cette dernière étape est le transfert d'embryon).

- FIV-GPA : Fécondation in vitro-Gestation pour autrui. L'enfant est conçu à partir des ovocytes de la mère intentionnelle, fécondés in vitro par le sperme du père intentionnel; les embryons ainsi obtenus sont replacés dans l'utérus de la gestatrice. Il n'existe donc aucun lien génétique entre l'enfant et la mère gestationnelle.

- Gestatrice, mère gestationnelle : femme portant un enfant qui sera élevé par autrui.

- GPA avec compensation financière : lorsque que la gestatrice est remboursée des frais liés à la grossesse et qu'elle reçoit une compensation financière au titre de la peine, du temps passé et des inconvénients liés à la grossesse.

- GPA sans compensation financière : lorsque seuls les frais liés à la grossesse sont pris en charge par les parents intentionnels.

- GPA : Gestation pour autrui. Fait pour une femme de porter un enfant qui sera élevé par d'autres personnes, en général un couple infertile.

- HLA-compatible : Human Leucocyt Antigen-compatible (HLA : antigènes des leucocytes humains).

- IA : Insémination artificielle. Technique d'assistance médicale à la procréation qui consiste à déposer le sperme dans l'appareil génital féminin, au niveau du vagin ou de l'utérus.

- IA-GPA : Insémination-Gestation pour autrui. L'enfant est conçu par insémination artificielle de la gestatrice avec le sperme du père intentionnel; la gestatrice est donc à la fois mère génétique et mère gestationnelle de l'enfant.

- IMG : Interruption médicale de grossesse.

- INSERM : Institut national de la santé et de la recherche médicale.

- IVG : Interruption volontaire de grossesse. 
- Maternité de substitution : gestation pour autrui. Cependant, ce terme réduit la maternité à la seule fonction de gestation, et d'autre part, le terme de substitution, appliqué en général à la gestatrice (désignée alors comme la mère de substitution) pourrait aussi bien s'appliquer à la mère intentionnelle.

- PMA : Procréation médicalement assistée. 